\title{
Optimization of Synthesis Conditions of Indigotindisulfonate Lithium Based on Orthogonal Experimental Design Method
}

\author{
Yu Jun ${ }^{1, *}$, Wanma Eri', Sun Shaorui ${ }^{2}$ \\ ${ }^{1}$ Institute of Chemistry \& Chemical Engineering, Qinghai University for Nationalities, Xining, China \\ ${ }^{2}$ Beijing Key Laboratory for Green Catalysis and Separation, College of Environmental and Energy Engineering, Beijing University of \\ Technology, Beijing, China
}

Email address:

yujuny@126.com (Yu Jun), 597737494@qq.com (W. Eri), sunsr@bjut.edu.cn (Sun Shaorui)

${ }^{*}$ Corresponding author

\section{To cite this article:}

Yu Jun, Wanma Eri, Sun Shaorui. Optimization of Synthesis Conditions of Indigotindisulfonate Lithium Based on Orthogonal Experimental Design Method. World Journal of Applied Chemistry. Vol. 5, No. 1, 2019, pp. 1-5. doi: 10.11648/j.wjac.20200501.11

Received: November 30, 2019; Accepted: December 25, 2019; Published: January 7, 2020

\begin{abstract}
With the developing research on anode materials for lithium ion batteries, organic anode materials have gained increasing attention nowadays. Compared with inorganic anode materials, organic anode materials have numerous material options and extensive sources. Moreover, they consume low energy and can enter carbon cycles, which are environment friendly. indigotindisulfonate lithium is characterized with a unique redox reaction and can be used as an anode material. Indigotindisulfonate Lithium can be produced by using indigo as a raw material sulfonated with fuming sulfuric acid, and further reacted with $\mathrm{Li}_{2} \mathrm{CO}_{3}$. Regarding this issue, impacts of various factors under different conditions were investigated, and an orthogonal experimental design method was proposed based on optimization conditions. This method was established through T-6 new century ultraviolet spectrophotometer to determine the influence of different conditions, and develop a best combination based on different microwave powers $(\mathrm{w})$, temperatures $\left({ }^{\circ} \mathrm{C}\right)$, and time lengths $(\mathrm{min})$. In this experiment, the maximum absorption peak was determined by scanning wave length, under which the absorbency was identified and the standard curve was established. In order to determine the optimized synthesis condition of indigotindisulfonate lithium, orthogonal experiment design method was applied combining three levels coupled with four factors. From this study, it is concluded that $550 \mathrm{w}, 3 \mathrm{~min}$, $30^{\circ} \mathrm{C}$ with an indigo-fuming sulfuric acid concentration ratio of $0.02: 1$ is the best condition to synthesize indigotindisulfonate lithium.
\end{abstract}

Keywords: Indigo, Fuming Sulfuric Acid, Orthogonal Test, Indigotindisulfonate Lithium

\section{Introduction}

With the development of science and technology, lithium and its compounds are more and more widely used in various fields. The demand of lithium products in the global world is increasing yearly, and the cost of lithium products is increasing significantly as well. There are abundant salt lake lithium resources in Qaidam basin of Qinghai province. The exploitation of lithium and its compounds will play a significant role in enhancing economy in Western part of China. Mounting evidence demonstrates that the resource oflithium brine in China ranks the top first in the global world, accounting for about $66 \%$ of the total lithium reserve in the salt lake. Moreover, the quantity of lithium chloride in Qarhan Salt Lake also ranks No.1 in the world [1].

There is abundant lithium resource in the Qaidam Salt Lake locating in the basin of Qinghai. Lithium is one of the most elementary raw materials in the production of battery. Lithium battery is very critical energy storage and has been widely applied in mobile communication equipment, mobile computing devices, portable power tools, electrical vehicles, emergency power supply and other systems. The cost and performance of lithium battery are closely related with the cathode material of lithium. At present, common inorganic anode materials including $\mathrm{LiCoO}_{2}, \mathrm{LiNiO}_{2}, \mathrm{LiMn}_{2} \mathrm{O}_{4}$ and $\mathrm{LiFePO}_{4}$, have been commercialized and deeply investigated. 
But those inorganic materials have two obvious shortcomings, one is limited resources for raw mineral materials (except Fe); the other is that those inorganic materials are not environment-friendly since their production and recycling require high temperature and high energy consumption, and release a large amount of $\mathrm{CO}_{2}$, leading to greenhouse effect, which is not in line with the concept of " Green, low carbon, and environment protection" in contemporary society.

In recent years, organic anode materials have gained increasing attention. Compared with inorganic anode materials, organic anode materials have numerous material options and extensive sources. Moreover, they consume low energy and can enter carbon cycles, which are environment-friendly. A plenty of organic anode materials have been discovered and reported in recent studies, indigotindisulfonate lithium is recently discovered as an outstanding organic anode material to make lithium ion battery, and can be used in multiple fields.

In this study, orthogonal experimental design method was used to optimize the synthesis conditions for indigotindisulfonate lithium. In recent years, Current scientific research is mainly focusing on inorganic materials composed of lithium, while the research on organic anode materials is relatively limited, and few mounting evidence can be accessed as references. In this study, the orthogonal design experiment method with 3 levels and 4 factors was established to optimize the condition of synthesis of indigotindisulfonate lithium. The main four factors are different reaction temperatures $\left({ }^{\circ} \mathrm{C}\right)$, time lengths (min), microwave powers (w), and material composition ratios. Various factors were studied to observe their impacts on synthesis of indigotindisulfonate lithium.

According to published literatures on indigotin disulfonate [2-4], the purpose of our experiment mainly focuses on the preparation of indigotin lithium dithionate. The yield of indigotindisulfonate lithium was investigated by optimizing different temperatures, concentrations and time lengths, and neutralization with $\mathrm{Li}_{2} \mathrm{CO}_{3}$.

\section{Experiment}

\subsection{Instruments and Reagents}

T-6 new century uv spectrophotometer, Xh-800s microwave hydrothermal parallel synthesis instrument, $250 \mathrm{~mL}$ flask, $50 \mathrm{~mL}$ measuring cylinder, $20 \mathrm{ml}$ pipette, $100 \mathrm{ml}$ flask, $1000 \mathrm{~mL}$ flask, $10 \mathrm{~mm}$ colorimeter.

The standard solution of indigotindisulfonate lithium: dissolve $2.0 \mathrm{~g}$ of indigotindisulfonate lithium in deionized water, and titrate the volume to $1000 \mathrm{~mL}$ as a reserve solution.

All reagents used in this experiment were analytically pure and water was deionized water.

\subsection{Experiment Theory}

The indigotindisulfonate lithium was synthesized by using indigo as a raw material. Indigo solution with concentrations of $0.02 \mathrm{~g} / \mathrm{L}, 0.2 \mathrm{~g} / \mathrm{L}$ and $2.0 \mathrm{~g} / \mathrm{L}$ were mixed with $98 \%$ fuming sulfuric acid. Under different microwave powers, temperatures, time lengths, the mixtures were further reacted with sufficient $\mathrm{Li}_{2} \mathrm{CO}_{3}$, the indigotindisulfonate lithium was synthesized and purified. The yield of indigotindisulfonate lithium was determined by the absorbance [5-11].

\subsection{Experimental Methods}

Analytical testing technology is a comprehensive scientific research method with interdisciplinary development, frontier application and integration of chemistry, physics and other disciplines, which mainly studies the compositions, states and structures of different substances. It also plays an indispensable role in scientific research especially for other disciplines to obtain relevant chemical information. In this study, T-6 new century uv spectrophotometer was applied to scan in the wavelength range between $320 \mathrm{~nm}$ to $880 \mathrm{~nm}$ first, then condition was set by using the wavelength under the maximum absorption peak.

The orthogonal experiment design method with multiple factors and levels was adopted to select representative points from the comprehensive experiment in order to reduce the redundant experiments. Those points were evenly distributed and nicely uniformed.

Indigo with different amount of $0.02 \mathrm{~g}, 0.2 \mathrm{~g}$ and $2.0 \mathrm{~g}$ were respectively dissolved in $1000 \mathrm{~mL}$ flask with purified water, they were stirred and shaken continuously to make them completely dissolved.

Next, $10 \mathrm{~mL}$ of prepared indigo solution was mixed with $10 \mathrm{~mL}$ fuming sulfuric acid in different reaction temperatures $\left({ }^{\circ} \mathrm{C}\right)$, time lengths ( $\mathrm{min}$ ), microwave powers (w), and ratios of materials to obtain the indigotin lithium disulfonic acid. This mixture was then reacted with $2.5 \mathrm{~g}$ of lithium carbonate to obtain indigotindisulfonate lithium with continuous stirring and shaking, and was placed for $30 \mathrm{~min}$. Then the absorbance was measured.

\section{The Results and Analysis}

\subsection{Absorption Curve}

The deionized water was added to $10 \mathrm{~mL}$ of indigotindisulfonate lithium reserve solution to a total volume of $1000 \mathrm{~mL}$. The final concentration of this prepared solution was $20 \mathrm{ug} / \mathrm{L}$. T-6 new century uv spectrophotometer automatically scanned from the range of 320 to $880 \mathrm{~nm}$. The maximum absorption peak was selected from the absorption spectrum, which is shown in table 1.

Table 1. Absorbance at different wavelengths.

\begin{tabular}{llllll}
\hline $\boldsymbol{\lambda} \mathbf{n m}$ & $\mathbf{A}$ & $\boldsymbol{\lambda} \mathbf{n} \mathbf{m}$ & $\mathbf{A}$ & $\boldsymbol{\lambda} \mathbf{n m}$ & $\mathbf{A}$ \\
\hline 320 & 0.344 & 510 & 0.096 & 700 & 0.029 \\
330 & 0.297 & 520 & 0.114 & 710 & 0.026 \\
340 & 0.289 & 530 & 0.138 & 720 & 0.023 \\
350 & 0.241 & 540 & 0.174 & 730 & 0.022 \\
360 & 0.170 & 550 & 0.216 & 740 & 0.021 \\
370 & 0.110 & 560 & 0.266 & 750 & 0.021 \\
380 & 0.088 & 570 & 0.307 & 760 & 0.024 \\
390 & 0.069 & 580 & 0.358 & 770 & 0.024 \\
400 & 0.069 & 590 & 0.404 & 780 & 0.023 \\
410 & 0.075 & 600 & 0.455 & 790 & 0.023 \\
420 & 0.072 & 610 & 0.490 & 800 & 0.022 \\
\hline
\end{tabular}




\begin{tabular}{llllll}
\hline$\lambda / \mathbf{n m}$ & $\mathbf{A}$ & $\lambda / \mathbf{n m}$ & $\mathbf{A}$ & $\lambda / \mathbf{n m}$ & $\mathbf{A}$ \\
\hline 430 & 0.074 & 620 & 0.463 & 810 & 0.023 \\
440 & 0.074 & 630 & 0.378 & 820 & 0.022 \\
450 & 0.084 & 640 & 0.277 & 830 & 0.021 \\
460 & 0.078 & 650 & 0.187 & 840 & 0.021 \\
470 & 0.077 & 660 & 0.119 & 850 & 0.023 \\
480 & 0.074 & 670 & 0.070 & 860 & 0.024 \\
490 & 0.078 & 680 & 0.052 & 870 & 0.021 \\
500 & 0.082 & 690 & 0.035 & 880 & 0.020 \\
\hline
\end{tabular}

As shown in table 1, the maximum of absorption wavelength peak was at $610 \mathrm{~nm}$.

\subsection{The Establishment of Standard Curve}

Take $0.0 \mathrm{~mL}, 2.0 \mathrm{~mL}, 4.0 \mathrm{~mL}, 6.0 \mathrm{~mL}, 8.0 \mathrm{~mL}$ and $10.0 \mathrm{~mL}$ of solution from prepared $0.0202 \mathrm{~g} / \mathrm{L}$ indigotindisulfonate lithium solution, respectively. Each volume of solution was fixed to $10 \mathrm{~mL}$ with deionized water. The amount of indigotindisulfonate lithium was $0,4.02,8.08,12.12,16.16,20.2 \mu \mathrm{g} / \mathrm{mL}$, respectively. At the wavelength of $610 \mathrm{~nm}, 10 \mathrm{~mm}$ cuvette was used for photometric analysis. With distilled water as reference solution, the absorbance of the reaction solution was determined. The amount and absorbance were used as the abscissa and ordinate respectively to make the standard curve of indigotindisulfonate lithium solution. The result is shown in table 2 and Figure 1.

Table 2. Absorbance of different concentrations of Indigotindisulfonate Lithium solution.

\begin{tabular}{lllllll}
\hline $\begin{array}{l}\text { Concentration } \\
(\mu \mathrm{g} / \mathrm{mL})\end{array}$ & 0 & 4.02 & 8.08 & 12.12 & 16.16 & 20.2 \\
Absorbance & 0 & 0.112 & 0.214 & 0.312 & 0.385 & 0.487 \\
\hline
\end{tabular}

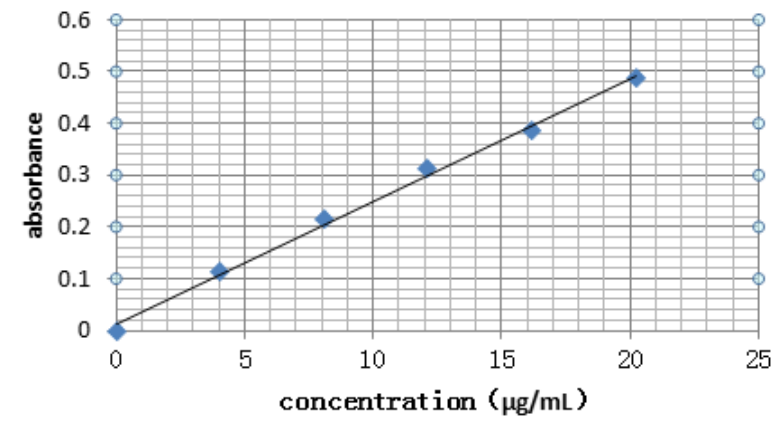

Figure 1. Standard working curve of Indigotindisulfonate Lithium solution.

\subsection{Synthesis Parameter of Indigotindisulfonate Lithium}

Orthogonal experimental design in experimental research is used for single-factor or two-factor tests, since it includes a few factors, and the design, implementation and analysis of the test are relatively simple. However, it is often necessary to examine three or more experimental factors at the same time in a real practice. If a comprehensive test is to be determined, the scale will be very huge, and it is often difficult due to the limitations of test conditions. Orthogonal experimental design is an efficient experimental design method to arrange multi-factor experiments which can be used to find an optimal combination [12-15].

The multi-factor analysis method of orthogonal experimental design was used to study and discuss different synthesis conditions of indigotindisulfonate lithium with different microwave powers $(\mathrm{w})$, temperatures $\left({ }^{\circ} \mathrm{C}\right)$, time lengths ( $\mathrm{min}$ ) and material composition ratios, which is indicated in table 3 and table 4.

Table 3. L9(3)4 Microwave oven synthesis [IC] Li meter head design.

\begin{tabular}{lllll}
\hline level & A & B & C & D \\
\hline & power $(\mathbf{w})$ & time (min) & temperature $\left({ }^{\circ} \mathbf{C}\right)$ & $\mathbf{C}_{(\mathbf{I C )})} \mathbf{C}_{(\text {oleum) }}$ \\
\hline 1 & 350 & 2 & 30 & $0.02: 1$ \\
2 & 550 & 3 & 40 & $0.2: 2$ \\
3 & 750 & 4 & 60 & $2.0: 4$ \\
\hline
\end{tabular}

Table 4. Experimental data and calculation analysis of conversion rate.

\begin{tabular}{|c|c|c|c|c|c|}
\hline NO. & $\mathbf{A}(\mathbf{W})$ & B (min) & $\mathrm{C}\left({ }^{\circ} \mathrm{C}\right)$ & D & [IDL]Li Productivity (\%) \\
\hline 1 & 1 & 1 & 1 & 1 & 31.9 \\
\hline 2 & 1 & 2 & 2 & 2 & 26.4 \\
\hline 3 & 1 & 3 & 3 & 3 & 22.95 \\
\hline 4 & 2 & 1 & 2 & 3 & 27.07 \\
\hline 5 & 2 & 2 & 3 & 1 & 38.5 \\
\hline 6 & 2 & 3 & 1 & 2 & 34.2 \\
\hline 7 & 3 & 1 & 3 & 2 & 20.29 \\
\hline 9 & 3 & 3 & 2 & 1 & 31.6 \\
\hline $\mathrm{K} 1$ & 81.25 & 79.26 & 98.8 & 102 & \\
\hline $\mathrm{K} 2$ & 99.77 & 97.6 & 85.07 & 80.89 & \\
\hline $\mathrm{K} 3$ & 84.59 & 88.75 & 81.74 & 82.72 & \\
\hline k1 & 27.08 & 26.42 & 32.93 & 34 & \\
\hline k2 & 33.26 & 32.53 & 28.36 & 26.96 & \\
\hline k3 & 28.2 & 29.58 & 27.25 & 27.57 & \\
\hline Order & $\mathrm{D}>\mathrm{A}>\mathrm{B}>\mathrm{C}$ & & & & \\
\hline Optimal levels & $\mathrm{A} 2$ & B2 & $\mathrm{C} 1$ & D1 & \\
\hline Optimal combination & A2 B2 C1 D1 & & & & \\
\hline
\end{tabular}




\subsection{Interpretation of Result}

The synthesis conditions of indigotindisulfonate lithium were determined in different microwave powers (w), temperatures (c), times ( $\mathrm{min}$ ) and material composition ratios. The optimum condition of was determined by orthogonal analysis.

In the Table 4, K1. K2. K3 are the sum of experimental results of each factor at the level of 1,2 and 3. k1, k2 and k3 are the average repeatability of $\mathrm{K} 1, \mathrm{~K} 2$ and $\mathrm{K} 3$. $\mathrm{R}$ is the range, which is the difference between the maximum and minimum value in $\mathrm{k} 1, \mathrm{k} 2$ and $\mathrm{k} 3$.

According to the order of range values in this table, the material composition ratios fluctuates the most under the selected absorption wavelength, which has a significant influence in this experiment. Thus, the material composition ratio is one of the most critical factors for optimization of indigotindisulfonate lithium.

The optimization of each factor is selected according to the ranges of extreme differences. After optimization of synthesis condition for indigotindisulfonate lithium, the best optimized combination is $\mathrm{A} 2 \mathrm{~B} 2 \mathrm{C} 1 \mathrm{D} 1$, which is $550 \mathrm{w}, 3 \mathrm{~min}, 30^{\circ} \mathrm{C}$ with an indigo solution - fuming sulfuric acid concentration ratio of 0.02:1.

From the range analysis in table $4, R_{D}>R_{A}>R_{B}>R_{C}$, that is to say, the material composition ratios has the greatest influence in optimization of synthesis of indigotindisulfonate lithium, followed with microwave powers, temperatures, and time lengths has the least impact on the optimization of synthesis of indigotindisulfonate lithium. The optimized condition is the combination of all horizontal linear factors, that is $\mathrm{A}_{2}, \mathrm{~B}_{2}, \mathrm{C}_{2}, \mathrm{D}_{2}$.

\section{Summary}

The orthogonal experimental design method was adopted in this experiment to determine the optimized synthesized condition of indigotindisulfonate lithium. The optimal condition was under $550 \mathrm{w}, 3 \mathrm{~min}, 30^{\circ} \mathrm{C}$ with a concentration ratio of 0.02:1 between indigo solution and fuming sulfuric acid.

It is scientific to arrange the test according to the orthogonal table, which can not only make the test points evenly distributed, but also reduce redundant tests, and simplify the calculation and analysis as well. From the perspective of test preparation, operation and the total cost, this method is simple, fast, precise, and cost-effective with a good repeatability.

Indigotindisulfonate lithium is known as an organic anode material, and has been applied in lithium ion battery research in recent years. With more researchbeing performed, and the electrochemical theory of non-aqueous system prominently developed, lithium ion battery has been widely used as clean electrical storage in communication fields military, aviation as well as other fields. As the crucial material of lithium ion battery, indigotindisulfonate lithium has a great potential in developing numerous types of lithium ion batteries, which will contribute the significant development of lithium ion battery industry in the future.

\section{Acknowledgements}

Yu Jun would like to acknowledge the financial support from Applied Basic Research Project of Qinghai Science and Technology Department (2017-ZJ-795). The authors acknowledge the experiment platform support from the Institute of Chemistry\&Chemical Engineering, Qinghai University for Nationalities, Xining, China.

\section{References}

[1] Gao Shi-yang. Exploitation of Lithiu in Salt Lakes and Environment. J Salt Lake Res, Vol. 8, No. 1, 2000, pp. 17-23.

[2] Chen Xiao. Indigo carmine removal by the biogenic Mn oxides and active carbon doped with Mn oxides. Huazhong Agricultural University, 2013.

[3] Huo jian-hong, Nie Jin-lian, Yang Shang-ming. Chemical Engineering \& Equipment, No. 3, 2013, pp. 167-169.

[4] Wang Wei, Chu Ben-li, Wang Jia-cheng, Zhou Liang-hua, He Song-hua. Preparation of Vanadium Substituted Magnetites and lts Efect on the Degradation of Indigo Carmine. GuangZhou Chemical Industry, Vol. 43, No. 6, 2015, pp. $59-61+91$.

[5] Bai Lin-shan, zhang Jin-hua, Zhang Shi-huan. Kinetic Spectrophotometric Determination of Vanadium in Steel Samples with Indige. Spectroscopy and Spectral Analysis, No. 1, 2002, pp. 120-122.

[6] Shen Jing, Huang Jian, Li Qian, Chen Wei-dong, Zheng Bi-fa. On Measurement of Ozone in the Air by Spectrophotometry of IDS. Sichuan Environment, Vol. 3, No. 1, 1998, pp. 78-81.

[7] Wang Yu-ping, Wang Juan. Calibration of indigo sodium disulfonate solution. Environmental monitoring management and technology, No. 2, 1994, pp. 39-42.

[8] Analysis room of shandong institute of soil analysis. Determination of NPK in organic fertilizers. Soil fertilizer, No. 4, 1975, pp. 47-49.

[9] Ran Guang-fen, Chen Yu-feng, Ma Hai-zhou, Xu Jian-xin, Gao Dong-lin, Meng Qing-fen. Determination of trace nitrate in Clcontaining mineral by spectrophotometry method. Inorganic Salt Industry, No. 10, 2006, pp. 57-59.

[10] Jiang Li-chun, Tang Shao-ming, You Qing, Wang Yue-hui, Peng Jun. Spectrophotometric Determinat ion of Ozone in Water by the Color2Fading Reaction of Sodium Indigo disulfonate. Physical and chemical inspection (chemical branch), Vol. 47, No. 2, 2011, pp. 180-182.

[11] Li Yin-huan, Li Hong-yan, Zhang Xiu-lan, Hu Fu-xin, Zhang Meng-meng. Study on Kinetics and Adsorption of Acid Blue onto Anion-Cation Organobentonite. Journal of xinyang normal university (natural science edition), Vol. 23, No. 2, 2010, pp. 284-287.

[12] Zhou Huaiwu, Ni Yongxing. Probability statistics of pharmaceutical application [M], Shanghai, Baijia Publishing House. 1990, pp. 235. 
[13] Yu Benming, Wang Zhongzhuang, Hu Jinhong. study on extraction and separation of curcumin from turmeric [J] pharmaceutical care and research. 2000, 6 (4), pp. 272-279.

[14] Liu Dingyuan. Methods of mathematical statistics in medicine [M], Beijing, People's medical publishing house, 1999, pp. 174-175.
[15] Zhu Guoqiang, Liu qingou. Methods of mathematical statistics in medicine $[\mathrm{M}]$. Beijing, higher education press, 2004, pp. 201-222. 